\title{
Magnetic fluctuations and resonant peak in cuprates: a microscopic theory
}

\author{
I. Sega ${ }^{1}$, P. Prelovšek ${ }^{1,2}$ and J. Bonča ${ }^{1,2}$ \\ ${ }^{1} J$. Stefan Institute, SI-1000 Ljubljana, Slovenia and \\ ${ }^{2}$ Faculty of Mathematics and Physics, University of Ljubljana, SI-1000 Ljubljana, Slovenia
}

(Dated: 28th October 2018)

\begin{abstract}
The theory for the dynamical spin susceptibility within the $t-J$ model is developed, as relevant for the resonant magnetic peak and normal-state magnetic response in superconducting (SC) cuprates. The analysis is based on the equations of motion for spins and the memory-function presentation of magnetic response where the main damping of the low-energy spin collective mode comes from the decay into fermionic degrees of freedom. It is shown that the damping function at low doping is closely related to the c-axis optical conductivity. The analysis reproduces doping-dependent features of the resonant magnetic scattering.
\end{abstract}

PACS numbers: 71.27.+a, 75.20.-g, 74.72.-h

Since its discovery in inelastic neutron scattering experiments in superconducting (SC) $\mathrm{YBa}_{2} \mathrm{Cu}_{3} \mathrm{O}_{7}$ [1], the magnetic resonance peak has been the subject of numerous experimental investigations as well as theoretical analyses and interpretations. The magnetic peak has been systematically followed in $\mathrm{YBa}_{2} \mathrm{Cu}_{3} \mathrm{O}_{6+x}$ (YBCO) into the underdoped regime

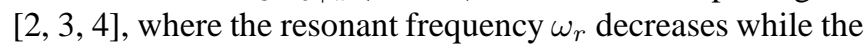
peak intensity is increasing. Its pronounced appearance is still related to the onset of SC, although it could start appearing even at $T>T_{c}$. More recent results confirm similar behavior in Bi2212 and T12201 cuprates [5].

Several theoretical hypotheses have been considered for the origin of the resonant peak: that it is a bound state in the electron-hole excitation spectrum [6], a consequence of a novel symmetry between antiferromagnetism (AFM) and SC [7] and that it represents collective spin-wave-like mode induced by strong AFM correlations [8, 9]. There is also an ongoing debate whether the resonant peak is intimately related to the mechanism of SC and whether it can account for anomalies in single-electron properties, as tested in angle resolved photoemission spectroscopy.

The scenario of a resonant mode as a collective magnetic mode seems to correspond well to experimental facts, in particular the qualitative development of the resonant mode with doping and its onset for $T<T_{c}$. Still the status of the theory of the resonant mode, and moreover of the magnetic response in cuprates in general, is not satisfactory, both from the point of understanding and of the appropriate analytical method. Relevant microscopic models, such as the Hubbard model and the $t-J$ model have been so far studied in the weak coupling or random-phase approximation [6], neglecting strong correlations. The latter have been considered using a Hubbard-operator technique [10], and more recently within the self-consistent slave-boson approach [11], self-consistent spin-fluctuation method [12], as well as within the phenomenological spin-fermion model [8, G].

Our aim is to develop a theory of the dynamical spin susceptibility $\chi_{\mathbf{q}}(\omega)$ within the $t-J$ model. The natural approach to analyse collective modes is the memory-function formalism [13]. In analogy to the previous study of spectral functions [14] we employ the method of equations of motion (EQM) to generate the spin dynamics and in particular to establish the effective decay of localized spins into fermionic degrees of freedom, which is the essential ingredient to describe the damping of the collective mode as well as the destruction of the long-range AFM order at finite doping.

We study the $t-J$ model

$$
H=-\sum_{i, j, s} t_{i j} \tilde{c}_{j s}^{\dagger} \tilde{c}_{i s}+J \sum_{\langle i j\rangle}\left(\mathbf{S}_{i} \cdot \mathbf{S}_{j}-\frac{1}{4} n_{i} n_{j}\right)
$$

on a square lattice. In order to be close to the physics in cuprates we take into account besides the nearest neighbor (n.n.) hopping $t_{i j}=t$ also the next n.n. $t_{i j}=t^{\prime}$ hopping. Strong correlations among electrons are incorporated via projected operators, e.g. $\tilde{c}_{i s}^{\dagger}=\left(1-n_{i,-s}\right) c_{i s}^{\dagger}$, which do not allow for double occupancy of sites, and in $J \ll t$ where for cuprates we assume furtheron $J=0.3 t$.

Within the memory function approach of Mori [13] to dynamical response functions, which we here pursue, the dynamical spin susceptibility $\chi_{\mathbf{q}}(\omega)$ can be expressed as

$$
\chi_{\mathbf{q}}(\omega)=\frac{-\eta_{\mathbf{q}}}{\omega^{2}+\omega M_{\mathbf{q}}(\omega)-\omega_{\mathbf{q}}^{2}},
$$

in which form it is particularly well suitable for the analysis of coherent collective magnetic response, as manifest in the resonant peak in cuprates. Here, $\omega_{\mathbf{q}}$ is related to the dispersion of the collective mode provided that the mode damping is small enough, i.e. $\gamma_{\mathbf{q}} \sim M_{\mathbf{q}}^{\prime \prime}\left(\omega_{\mathbf{q}}\right)<\omega_{\mathbf{q}}$. In the opposite case, we are dealing with an overdamped mode, as seems to be generally the case for the magnetic response near the AFM wavevector $\mathbf{q} \sim \mathbf{Q}=(\pi, \pi)$ in the normal state of cuprates.

In order to evaluate the quantities entering Eq.(2) we follow the formalism of memory functions [13], defining the scalar products and projections in terms of static response functions $(A \mid B)=\chi_{A B}^{0}=-\left\langle\left\langle A^{\dagger} ; B\right\rangle_{\omega=0}\right.$, and the action of the Liouville super-operator $\mathcal{L} A=[H, A]$. Within this framework we can expres

$$
\begin{gathered}
\left.\eta_{\mathbf{q}}=\left(\mathcal{L} S_{\mathbf{q}}^{z} \mid \mathcal{L} \quad S_{\mathbf{q}}^{z}\right)=\left\langle\left[S_{-\mathbf{q}}^{z} \mathcal{L} S_{\mathbf{q}}^{z}\right)\right]\right\rangle, \quad \omega_{\mathbf{q}}^{2}=\eta_{\mathbf{q}} / \chi_{\mathbf{q}}^{0}, \\
M_{\mathbf{q}}(\omega)=\left(\widetilde{Q} \mathcal{L}^{2} S_{\mathbf{q}}^{z}\left|\left[\mathcal{L}_{\widetilde{Q}}-\omega\right]^{-1}\right| \widetilde{Q} \mathcal{L}^{2} S_{\mathbf{q}}^{z}\right) / \eta_{\mathbf{q}}
\end{gathered}
$$


where $\mathcal{L}_{\widetilde{Q}}=\widetilde{Q} \mathcal{L} \widetilde{Q}$ is the projected Liouville super-operator, $\chi_{\mathbf{q}}^{0}=\chi_{\mathbf{q}}(\omega=0)$ is the static susceptibility and the projectors $\widetilde{Q}=Q^{\prime} Q$ are given by $\left.Q=1-\mid S_{\mathbf{q}}^{z}\right)\left[\chi_{\mathbf{q}}^{0}\right]^{-1}\left(S_{\mathbf{q}}^{z} \mid\right.$ and $Q^{\prime}=$ $\left.\left.1-\mid \mathcal{L} S_{\mathbf{q}}^{z}\right)\right)\left[\eta_{\mathbf{q}}\right]^{-1}\left(\mathcal{L} S_{\mathbf{q}}^{z} \mid\right.$, respectively.

In order to proceed we write down equations of motion (EQM) for the spin operators $S_{\mathbf{q}}^{z}$. By evaluating $\mathcal{L} S_{\mathbf{q}}^{z}$ it is straightforward to explicitly express $\eta_{\mathbf{q}}$ in Eq.(3) as

$$
\begin{aligned}
\eta_{\mathbf{q}} & =\frac{1}{4 N} \sum_{\mathbf{k}, s}\left[\epsilon_{\mathbf{q}+\mathbf{k}}^{0}+\epsilon_{\mathbf{q}-\mathbf{k}}^{0}-2 \epsilon_{\mathbf{k}}^{0}\right]\left\langle\tilde{c}_{\mathbf{k} s}^{\dagger} \tilde{c}_{\mathbf{k} s}\right\rangle \\
& +\frac{1}{2 N} \sum_{\mathbf{k}}\left[J_{\mathbf{q}+\mathbf{k}}+J_{\mathbf{q}-\mathbf{k}}-2 J_{\mathbf{k}}\right]\left\langle S_{\mathbf{k}}^{+} S_{-\mathbf{k}}^{-}\right\rangle,
\end{aligned}
$$

where $\epsilon_{\mathbf{k}}^{0}$ is the 'free' band dispersion following from tightbinding hopping $t_{i j}$. We are interested in the vicinity $\mathbf{q} \sim \mathbf{Q}$. We note that $\eta_{\mathbf{q}}$ is closely related to the internal energy, i.e. $\eta_{\mathbf{Q}} \sim-\langle H\rangle$. This indicates that $\eta_{\mathbf{q}}$ is not strongly dependent neither on temperature $T$ nor on $\mathbf{q}$, while the doping dependence $c_{h}=1-\left\langle n_{i}\right\rangle$ (at low doping) $\eta \sim a c_{h}|t|+b J$ is as well modest, where $a \sim b \sim 1$, as inferred e.g. from numerical studies of the model.

The evaluation of $\mathcal{L}^{2} S_{\mathbf{q}}^{z}$ is also straightforward, but more tedious. It is convenient to separate the action $\mathcal{L}^{2}=\mathcal{L}_{t}^{2}+$ $\mathcal{L}_{I}^{2}+\mathcal{L}_{J}^{2}$ with $\mathcal{L}_{I}^{2}=\left[\mathcal{L}_{t}, \mathcal{L}_{J}\right]_{+}$, involving different powers of kinetic and exchange terms, respectively. In the following analysis we assume that for the damping function $\Gamma_{\mathbf{q}}(\omega)=$ $M_{\mathbf{q}}^{\prime \prime}(\omega)$ at $\mathbf{q} \sim \mathbf{Q}$, at low $T \sim 0$ and in the doping regime of interest, i.e. of low to intermediate doping, the essential term is $\mathcal{L}_{t}^{2}$. Namely, we presume that at least in the normal state the damping $\Gamma_{\mathbf{q}}(\omega)$ approaches a constant $\gamma_{\mathbf{q}}$ for $\omega \rightarrow 0$ as in a Fermi liquid (although anomalous). Such a damping can arise only from the coupling to fermionic degrees of freedom, which then has to involve $H_{t}$ term.

One can give more arguments in support of our assumption. In an undoped system - AFM, only $\mathcal{L}_{J}^{2}$ is effective. However, it is well known that at $T=0$ such a term gives a damping $\gamma_{\mathbf{q}} \propto \tilde{q}^{2}$ where $\tilde{\mathbf{q}}=\mathbf{q}-\mathbf{Q}$, as well as $\Gamma_{\mathbf{Q}}(\omega) \propto \omega^{2}[15]$. Hence it leads to underdamped and well defined AFM magnon excitations for $\tilde{q} \rightarrow 0$, the origin being in the phase space of low-lying spin excitations restricted to $\tilde{q} \sim 0$. The argument about the $\mathcal{L}_{J}^{2}$ term can be extended to a doped system. Assuming the Fermi liquid form $\chi_{\mathbf{q}}^{\prime \prime}(\omega) \propto \omega$ a mode-coupling treatment here would again yield $\Gamma_{\mathbf{q}}(\omega) \propto \omega^{2}$. The role of $\mathcal{L}_{I}^{2}$, on the other hand, cannot be apriori neglected at $\omega \rightarrow 0$. However, within the same decoupling described below we get a vanishing contribution of diagonal $\mathcal{L}_{I}^{2} S_{\mathbf{q}}^{z}$ to $M_{\mathbf{q}}(\omega)$.

We should stress that the evaluation of $\mathcal{L}_{t}^{2} S_{\mathbf{q}}^{z}$ requires explicit consideration of the projections of fermionic operators in $H_{t}$. In the site-representation we can express

$$
\begin{gathered}
\mathcal{L}_{t}^{2} \mathbf{S}_{j}=-\sum_{k} t_{j k}^{2}\left(\mathbf{S}_{j}-\mathbf{S}_{k}\right) \mathcal{P}_{j k}+ \\
+\sum_{k l s} t_{j k}\left[t_{k l} \mathbf{S}_{j} \mathcal{T}_{j k} \tilde{c}_{j s}^{\dagger} \tilde{c}_{l s}-t_{j l} \mathcal{T}_{j l} \tilde{c}_{l s}^{\dagger} \tilde{c}_{k s} \mathbf{S}_{k}\right]+\text { H.c. }
\end{gathered}
$$

Here $\mathcal{T}_{i j}=n_{i}\left(1-n_{j}\right)+\mathcal{P}_{i j}$ and $\mathcal{P}_{i j}=n_{i} n_{j} / 2+2 \mathbf{S}_{i} \cdot \mathbf{S}_{j}$ is the spin-interchange operator. Complicated form of Eq.(5) reflects the well-known involved nature of correlated hopping in a strongly correlated system, i.e. with a reshuffling of spins along the hole path. Since the main goal is to get the coupling to nonlocal fermionic degrees, in EQM we replace operators $\mathcal{P}_{i j}, \mathcal{T}_{i j}$ by their thermal averages $P_{i j}, T_{i j}$, respectively, leading to an effective hopping renormalization in Eq.(5). Note that for the n.n. hopping, $T_{1}$ represents essential reduction, since in a Neél state one would get $P_{1}=0$ and only in heavily doped system $P_{1} \sim\left(1-c_{h}\right)^{2} / 2$. For the regime of interest, i.e., $0.1<c_{h}<0.25$ one can on the basis of numerical results for the $t-J$ model simplify $T_{1} \sim c_{h}$. We have to apply to Eq. (5) also the projector $\widetilde{Q}$. To the lowest order this implies that the projected operator does not contain explicitly the initial operator $S_{\mathbf{q}}^{z}$ itself. So we get

$$
\begin{gathered}
\widetilde{Q} \mathcal{L}_{t}^{2} S_{\mathbf{q}}^{z} \sim \frac{1}{2 \sqrt{N}} \sum_{\mathbf{k} s} w_{\mathbf{k q}} s \tilde{c}_{\mathbf{k s} s}^{\dagger} \tilde{c}_{\mathbf{k}+\mathbf{q}, s}, \\
w_{\mathbf{k q}}=\left(\epsilon_{\mathbf{k}}^{0}-\epsilon_{\mathbf{k}+\mathbf{q}}^{0}\right)\left(\tilde{\epsilon}_{\mathbf{k}}^{0}-\tilde{\epsilon}_{\mathbf{k}+\mathbf{q}}^{0}\right)-\zeta_{\mathbf{q}},
\end{gathered}
$$

where $\tilde{\epsilon}_{\mathbf{k}}^{0}$ is defined with renormalized hopping parameters $\tilde{t}_{i j} \approx t_{i j} T_{i j}$ whereas $\zeta_{\mathbf{q}}$ is determined by the condition $\sum_{\mathbf{k}} w_{\mathbf{k q}}=0$. We note that within an analogous approximation $\mathcal{L}_{I}^{2} S_{\mathbf{q}}^{z}=0$.

Eq.(6) represents a decay of spin variables into fermions in a doped system, so performing a decoupling (in a normal state) we get for the damping in the lowest approximation

$$
\Gamma_{\mathbf{q}}(\omega)=\frac{1}{2 \eta_{\mathbf{q}}} \int \frac{d \omega^{\prime}}{\omega}\left[f\left(\omega-\omega^{\prime}\right)-f\left(\omega^{\prime}\right)\right] R_{\mathbf{q}}\left(\omega, \omega^{\prime}\right),
$$

with

$$
R_{\mathbf{q}}\left(\omega, \omega^{\prime}\right)=\frac{\pi}{N} \sum_{\mathbf{k}} w_{\mathbf{k q}}^{2} A_{\mathbf{k}}\left(\omega^{\prime}\right) A_{\mathbf{k}+\mathbf{q}}\left(\omega-\omega^{\prime}\right),
$$

where $A_{\mathbf{k}}(\omega)$ are electron spectral functions. At low doping an alternative decoupling of fermionic operators directly in the site representation, Eq. (5), neglecting the coherence between different sites might be more appropriate, considering the fact that in underdoped systems spectral functions exhibit pronounced incoherent behavior. This would yield

$$
\widetilde{R}_{\mathbf{q}}\left(\omega, \omega^{\prime}\right) \approx \pi \zeta_{\mathbf{q}}^{2} \mathcal{N}\left(\omega^{\prime}\right) \mathcal{N}\left(\omega-\omega^{\prime}\right),
$$

where $\mathcal{N}(\omega)=(2 / N) \sum_{\mathbf{k}} A_{\mathbf{k}}(\omega)$ is the electron density of states (DOS). The form \&, @pis particularly appealing since by Eq. (17) the damping becomes proportional to the c-axis conductivity, i.e. $\Gamma_{\mathbf{q}}(\omega) \propto \sigma_{c}(\omega)$, which can be well represented within the same form [16].

Before proceeding to discussion of $\Gamma_{\mathbf{q}}(\omega)$ we note that the theory requires an additional input, i.e. in Eq.(2) we need either $\omega_{\mathbf{q}}$ or $\chi_{\mathbf{q}}^{0}$. A possibility is to fix unknowns with the sum rule in the paramagnetic phase

$$
\frac{1}{\pi} \int_{0}^{\infty} d \omega \operatorname{cth} \frac{\omega}{2 T} \chi_{\mathbf{q}}^{\prime \prime}(\omega)=\left\langle S_{-\mathbf{q}}^{z} S_{\mathbf{q}}^{z}\right\rangle=C_{\mathbf{q}}
$$

where in addition $(1 / N) \sum_{\mathbf{q}} C_{\mathbf{q}}=\left(1-c_{h}\right) / 4$. Static correlation functions $C_{\mathbf{q}}$ are rather well known within the $t-J$ model [17], in particular one can express $C_{\mathbf{Q}} \propto \xi^{2}$ where $\xi$ is the AFM correlation length, determined also experimentally in $\mathrm{La}_{2-x} \mathrm{Sr}_{x} \mathrm{CO}_{4}$ [18], with $\xi \propto 1 / \sqrt{c_{h}}$. 
In the present study we assume some simple forms for spectral functions $A_{\mathbf{k}}(\omega)$ although there exists also an analogous approach to fermion dynamics, yielding as well pseudogap (PG) features in $A_{\mathbf{k}}(\omega)$ from the coupling to spin degrees [14]. The simplest assumption in the normal state is to insert some effective coherent band crossing the Fermi energy, i.e. $A_{\mathbf{k}}(\omega) \sim Z_{\mathbf{k}} \delta\left(\omega-\epsilon_{\mathbf{k}}^{e f f}\right)$. Such a form yields at $T \rightarrow 0 \Gamma_{\mathbf{Q}}(\omega \rightarrow 0) \sim$ const from $R$, Eq.(8), as well as from expression (9), provided that the Fermi surface crosses the AFM zone boundary. The doping dependence enters $\Gamma$ in several ways. First, there should be an overall proportionality to $c_{h}$ which is evident from the relation of $\Gamma$ with $\sigma_{c}$. In an effective band picture the latter can arise from vanishing QP weight $\bar{Z} \propto \sqrt{c_{h}}$ assuming that the effective band width $\widetilde{W} \sim \tilde{a} c_{h}|t|+\tilde{b} J$ does not vanish for $c_{h} \rightarrow 0$. $\eta$ and $T_{i j}$ are less $c_{h}$-dependent in the regime of interest. We can estimate the size of normal-state damping as $\gamma_{\mathbf{Q}} \sim \pi \zeta_{\mathbf{Q}}^{2} \bar{Z}^{2} / 2 \widetilde{W}^{2} \eta_{\mathbf{Q}}$, which at low doping can be quite small $\gamma_{\mathbf{Q}} \ll t$. Nevertheless, from available numerical data we estimate $\gamma_{\mathbf{Q}}$ still too large for an underdamped collective mode at $\omega_{\mathbf{Q}}$ to exist.

In order to get un underdamped resonant mode at $\omega_{r}$ one needs a depleted damping $\Gamma_{\mathbf{Q}}\left(\omega_{r}\right)$. The latter can evidently arise in the SC state, $T<T_{c}$, from the SC gap. We can model this by introducing into Eqs. (8.9) an effective d-wave gap $\Delta_{\mathbf{k}}=\Delta_{0}\left(\cos k_{x}-\cos k_{y}\right) / 2$ via $A_{\mathbf{k}}(\omega)$ and $\mathcal{N}(\omega)$. Due to the broken symmetry we include in Eq. (8) also the anomalous spectral functions $F_{\mathbf{k}}(\omega)$ [8]. The SC gap eventually leads to the vanishing of $\Gamma_{\mathbf{Q}}\left(\omega<\omega_{\mathbf{Q}}^{*}\right)=0$ where $\omega_{\mathbf{Q}}^{*} \sim 2 \Delta_{\mathbf{k}^{*}}<2 \Delta_{0}$ while $\mathbf{k}^{*}$ is the position of the 'hot spot' along the AFM zone boundary.

Such an analysis with a SC d-wave gap is particularly appropriate for the situation close to optimum doping. Results for $\Gamma_{\mathbf{q}}(\omega)$ for several $\mathbf{q}$ along the zone diagonal are presented in Fig. 1a. Parameters are chosen so as to correspond to optimum doping $c_{h} \sim 0.2$, i.e. effective band with $\tilde{t}=0.3 t$, $\tilde{t}^{\prime}=-0.1 t, \bar{Z}=0.4$, and we take $\Delta_{0} \sim 0.1 t$. We note that the damping with a single step at $\mathbf{q}=\mathbf{Q}$ develops two steps for $\mathbf{q} \neq \mathbf{Q}$ and the threshold $\omega_{\mathbf{q}}^{*} \rightarrow 0$ closes for $\tilde{q}=|\mathbf{q}-\mathbf{Q}|>$ $q^{*} \sim 0.3$ [8]. This is a mechanism for the onset of strong damping of the collective mode for $\tilde{q}>q^{*}$ and its disappearance as the resonant character emerges. Another important feature is the 'normal state' damping $\gamma_{\mathbf{Q}}=\Gamma_{\mathbf{Q}}\left(\omega>\omega_{\mathbf{Q}}^{*}\right)$ calculated in this approach explicitly. We note that $\gamma_{\mathbf{Q}} \gg \omega_{r}$, preventing any coherent feature in the normal state.

The corresponding $\chi_{\mathbf{q}}^{\prime \prime}(\omega)$ is presented in Fig. 1b. We choose $\omega_{\mathbf{q}} \sim 0.38 t$ such as to yield $C_{\mathbf{q}} \sim 0.4$, being consistent with results within the $t-J$ model for intermediate doping [17]. At $\mathbf{q}=\mathbf{Q}$ the resonant mode is undamped since $2 \Delta_{0}>\omega_{r}$. It should be stressed, however, that due to quite large $\Gamma_{\mathbf{Q}}\left(\omega>\omega_{\mathbf{Q}}^{*}\right)$ the resonant frequency is significantly renormalized

$$
\omega_{r}=\omega_{\mathbf{Q}}\left[1+M_{\mathbf{Q}}^{\prime}(\omega) /\left.\omega\right|_{\omega=\omega_{r}}\right]^{-1 / 2}
$$

while its intensity is also reduced $I_{\mathbf{Q}} \sim C_{\mathbf{Q}} \omega_{r} / \omega_{\mathbf{Q}}$. The rest of the spectral weight is distributed over a shallow but very

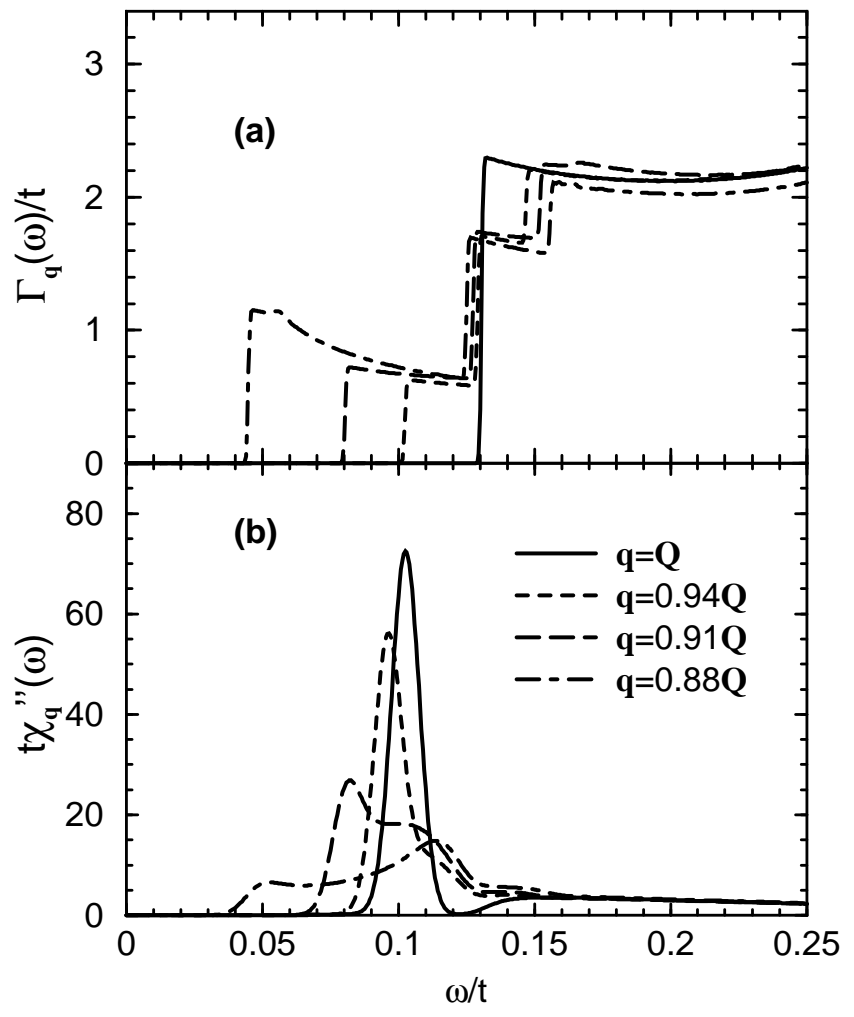

Figure 1: (a) Damping function $\Gamma_{\mathbf{q}}(\omega)$ at optimal doping $c_{h} \sim 0.2$ for various $\mathbf{q} \| \mathbf{Q}$ for a d-wave $\mathrm{SC}$, (b) corresponding spin response $\chi_{\mathbf{q}}^{\prime \prime}(\omega)$. Note that $t \sim 400 \mathrm{meV}$. The resonance peaks are artificially broadened with $\delta=0.01 t$.

broad continuum. On the other hand, moving away from $\mathbf{Q}$ the mode gets overdamped and merges with a broad continuum for $\tilde{q}>q^{*}$. In Fig. 1b we as well observe a downward dispersion of the resonant peak consistent with experiments [19, 20].

Analysing the regime of low doping it appears more appropriate to use the incoherent approximation, Eq.(9). It is crucial that the normal-state damping $\gamma_{\mathbf{Q}}$ also decreases with doping, scaling approximately as $\propto c_{h}$. On the other hand, it is rather clear that for underdoped cuprates the experimental data in the SC phase cannot be explained with a single gap only. Neutron scattering results for $\chi_{\mathbf{Q}}^{\prime \prime}(\omega)$ in the underdoped YBCO [2] indicate on the appearance of the resonance at $\omega_{r}$ at $T<T_{c}$, possibly even at $T>T_{c}$ [ [3]. However, in contrast to optimum doping the resonant mode at $\omega_{r}$ is quite damped. At the same time, the pronounced shoulder below the resonance, i.e. $\omega_{c}<\omega_{r}$, appears [2]. The drop in $\chi_{\mathbf{Q}}^{\prime \prime}\left(\omega<\omega_{c}\right)$ can be again interpreted with a coherent SC gap in $\Gamma_{\mathbf{Q}}(\omega)$, but with substantially diminished gap $2 \Delta_{c}<\omega_{r}$. Since the 'normal' damping is still too large, i.e. $\gamma_{\mathbf{Q}}>\omega_{r}$, we need to assume also the appearance of a pseudogap in DOS below $\omega \sim \omega_{p}$ for $T<T^{*}$ with $T^{*} \geq T_{c}$. This is well consistent with the behavior of $\sigma_{c}(\omega)$ in underdoped cuprates [21] where the pseudogap appears at $T<T^{*}$. In Fig. 2a we present characteristic $\Gamma_{\mathbf{Q}}(\omega)$, calculated at $T \sim 0$ for $c_{h} \sim 0.1$ 


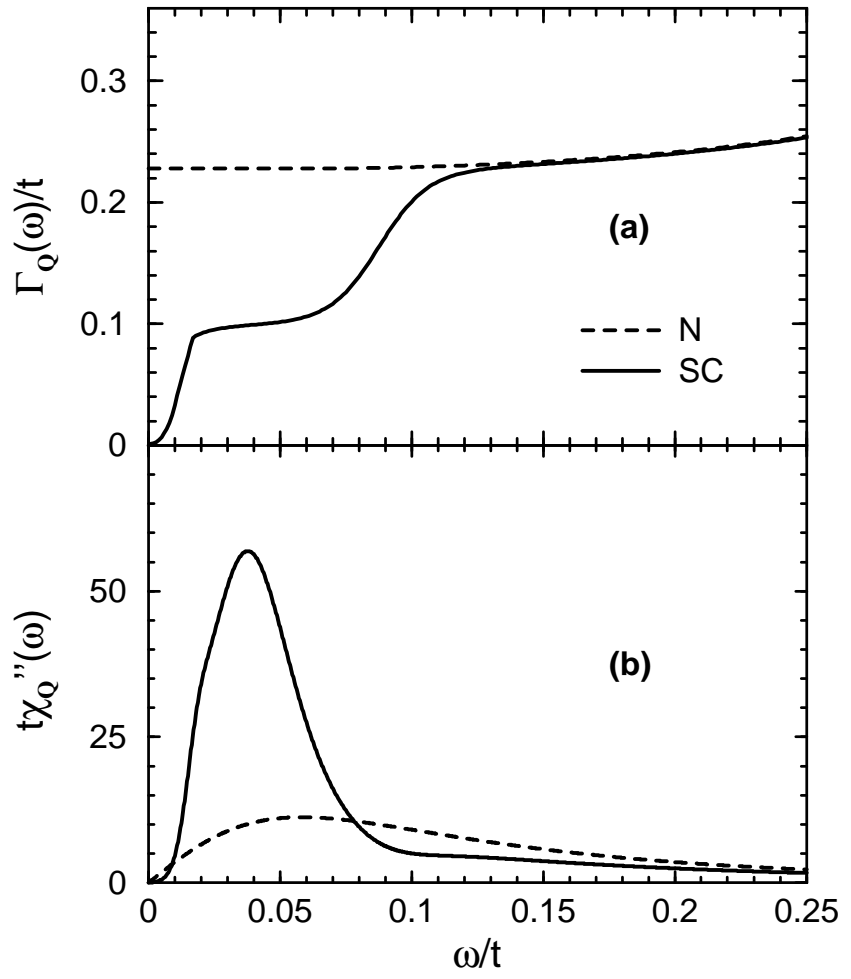

Figure 2: (a) $\Gamma_{\mathbf{Q}}(\omega)$ for underdoped $c_{h} \sim 0.1$. Dashed curve: normal state regime, $T \sim T^{*} \sim 0.05 t$. Full curve: SC regime. Here damping is assumed proportional to $\sigma_{c}(\omega)$ with an SC gap added below $\omega_{c} \sim 0.01 t$. (b) Corresponding $\chi_{\mathbf{Q}}^{\prime \prime}(\omega)$.

with corresponding $\Delta \sim 0.01 t$ and calculated $\eta_{\mathbf{Q}} \sim 0.25 t$ whereas $t \mathcal{N}\left(\omega>\omega_{p}\right) \sim 0.15$, as inferred from numerical results for the $t-J$ model [22]. An additional PG reduction for $\omega<\omega_{p} \sim 0.1 t$ is assumed, consistent with experimental data [21] together with an estimate of the DOS within the pseudogap $t \mathcal{N}\left(\omega<\omega_{p}\right) \sim 0.1$ [22]. The resulting $\chi_{\mathbf{Q}}^{\prime \prime}(\omega)$, corresponding to $C_{\mathbf{Q}} \sim 1.0$, is shown in Fig. 2 b. In the normal state $\chi_{\mathbf{Q}}^{\prime \prime}(\omega)$ is overdamped. Still, a substantial part of the sum rule is exhausted in the window $\omega<\omega_{\mathbf{Q}}$. It should be also pointed out that in the normal state the role of $T>0$ is essential via the sum rule 10 leading to $\omega_{\mathbf{Q}}(T)$ shifting with $T$. From Fig. $2 b$ it is evident that also in the SC state several features are different when compared to larger doping in Fig. 1b: a) The (spin) gap shoulder appears for $\omega<\omega_{c}$ below which there are no spin excitations. b) The resonant peak is damped even for $T<T_{c}$, but still underdamped. c) The spin response and the sum rule for $\chi_{\mathbf{Q}}^{\prime \prime}(\omega)$ are nearly exhausted within $\omega<\omega_{p}$. Since in the underdoped regime 'normal' $\gamma_{\mathbf{Q}}$ is also reduced, peak position given by Eq.(11) is not significantly renormalized and $\omega_{r} \sim \omega_{\mathbf{Q}}$. Consequently, for the underdamped mode using the sum rule, we obtain $\omega_{r} \sim \eta / C_{\mathbf{Q}} \propto 1 / \xi^{2} \propto c_{h}$.

To summarize, our analysis of the dynamical spin response and resonance peak in cuprates within the memory function approach can qualitatively, and at low doping even quantitatively, reproduce the spectra as measured in neutron scattering experiments [1], 2, 3, 斥. The central point of the present theory is the evaluation of the damping function $\Gamma_{\mathbf{q}}(\omega)$, using the EQM and considering the decay of spin fluctuation into electron-hole excitations as the dominant process. In the normal state we obtain a large damping $\gamma_{\mathbf{Q}}>\omega_{\mathbf{Q}}$ increasing with doping, leading generally to an overdamped AFM collective mode. Still it is important to realize that the renormalization due to $T_{i j} \ll 1$ in Eq.(6) is essential. Namely, without this reduction the damping would be much too large and in particular it would prevent the matching of the sum rule Eq. (10) with any pronounced short range AFM order $C_{\mathbf{Q}} \gg 1 / 4$.

Addressing the resonance peak in the SC state, we find that in optimally doped samples it can arise only inside the frequency gap $\sim 2 \Delta_{0}$, being strongly renormalized in comparison to the characteristic frequency $\omega_{\mathbf{Q}}$ and also reduced in the intensity. On the other hand the incoherent part of spin fluctuations extends over a broad frequency range $\sim \widetilde{W}$ and for the chosen parameters accounts for $\sim 80 \%$ of the integrated intensity $\int \chi_{\mathbf{Q}}^{\prime \prime}(\omega) d \omega$. In the underdoped (weakly doped) case, however, the sum rule at $T=0$ is almost completely exhausted within the peak width. As a consequence we obtain in this regime $\omega_{r} \propto c_{h}$, quite consistent with experiments [2]]. Moreover, in contrast to optimum doping with a single SC gap, in underdoped samples two scales seem to play the role in the SC state. The proportionality of damping to $\sigma_{c}$ would then indicate a gradual closing of the pseudogap leading to a crossover into an overdamped situation. More detailed analysis will be presented elsewhere.

Authors acknowledge the support of the Ministry of Education, Science and Sport of Slovenia.

[1] J. Rossat-Mignod et al., Physica C 185 - 189, 86 (1991).

[2] P. Bourges, in The Gap Symmetry and Fluctuations in High Temperature Superconductors, Ed. J. Bok et al. (Plenum Press, 1998).

[3] P. Dai et al., Science 284, 1344 (1999).

[4] H. F. Fong et al., Phys. Rev. B 61, 14773 (2000).

[5] H. F. Fong et al., Nature 398, 588 (1999); H. He et al., Science 295, 1045 (2002).

[6] M. Lavagna and G. Stemmann, Phys. Rev. B 49, 4235 (1994); D. Z. Liu, Y. Zha, and K. Levin, Phys. Rev. Lett. 75, 4130 (1995); I. I. Mazin and V. M. Yakovenko, Phys. Rev. Lett. 75, 4134 (1995).

[7] E. Demler and S. -C. Zhang, Phys. Rev. Lett. 75, 4126 (1995).

[8] D. K. Morr and D. Pines, Phys. Rev. Lett. 81, 1086 (1998).

[9] A. Abanov and A. V. Chubukov, Phys. Rev. Lett. 83, 1652 (1999).

[10] F. Onufrieva and J. Rossat-Mignod, Phys. Rev. B 52, 7572 (1995).

[11] J. Brinckmann and P. A. Lee, Phys. Rev. Lett. 82, 2915 (1999); Phys. Rev. B 65, 014502 (2001).

[12] T. Dahm, D. Manske, and L. Tewordt, Phys. Rev. B 58, 12454 (1998).

[13] H. Mori, Prog. Theor. Phys. 33, 423 (1965).

[14] P. Prelovšek and A. Ramšak, Phys. Rev. B 63, 180506(R) (2001); Phys. Rev. B 65, 174529 (2002). 
[15] T. Becher and G. Reiter, Phys. Rev. Lett. 63, 1004 (1989).

[16] P. Prelovšek, A. Ramšak, and I. Sega, Phys. Rev. Lett. 81, 3745 (1998).

[17] R. R. P. Singh and R. L. Glenister, Phys. Rev. B 46, 11871 (1992).

[18] R. J. Birgeneau et al., Phys. Rev. B 38, 6614 (1988).

[19] P. Bourges et al., Science 288, 1234 (1999).
[20] A. V. Chubukov, B. Jankó, and O. Tchernyshyov, Phys. Rev. B 63,180507 (2001).

[21] K. Tamasaku et al., Phys. Rev. Lett. 72, 3088 (1994); C. C. Homes et al., Physica C 254, 265 (1995).

[22] J. Jaklič and P. Prelovšek, Adv. Phys. 49, 1 (2000). 